\title{
Side population in MDA-MB-231 human breast cancer cells exhibits cancer stem cell-like properties without higher bone-metastatic potential
}

\author{
TORU HIRAGA ${ }^{1}$, SUSUMU ITO $^{2}$ and HIROAKI NAKAMURA ${ }^{1}$ \\ ${ }^{1}$ Department of Histology and Cell Biology, Matsumoto Dental University, 1780 Gobara-Hirooka, Shiojiri, \\ Nagano 399-0781; ${ }^{2}$ Division of Instrumental Analysis, Research Center for Human and Environmental Sciences, \\ Shinshu University, 3-1-1 Asahi, Matsumoto, Nagano 390-8621, Japan
}

Received August 5, 2010; Accepted September 21, 2010

DOI: 10.3892/or_00001073

\begin{abstract}
An increasing body of evidence suggests that cancers contain a small subset of their own stem-like cells called cancer stem cells (CSCs), which play critical roles in the initiation, maintenance and relapse of tumors. However, the role of CSCs in cancer metastasis, especially in metastasis to bone, has not been extensively studied. Side population (SP) has been shown to enrich CSCs in several types of cancer, including breast cancer. In the present study, we characterized the SP cells isolated from the human breast cancer cell line MDA-MB-231 in comparison to non-SP (NSP). Fluorescenceactivated cell sorter analysis demonstrated the existence of SP in MDA-MB-231 cells, which was markedly reduced in the presence of fumitremorgin $\mathrm{C}$, a specific inhibitor of ATP-binding cassette sub-family G member 2 (ABCG2). Quantitative RT-PCR analysis showed that ABCG2 mRNA expression was significantly higher in SP cells than in NSP cells. SP cells formed increased numbers of tumor-spheres in suspension culture. Furthermore, the tumor growth in the orthotopic mammary fat pad in nude mice was significantly accelerated in SP cells. On the other hand, the development of bone metastases determined by intracardiac injection into nude mice showed no difference between SP and NSP cells. SP abundance in the tumor cells isolated from the bone metastases was not increased either compared with that from the mammary tumors. These results suggest that the SP in MDA-MB-231 cells possesses some of the CSC-like properties but does not have higher metastatic potential to bone.
\end{abstract}

Correspondence to: Dr Toru Hiraga, Department of Histology and Cell Biology, Matsumoto Dental University, 1780 Gobara-Hirooka, Shiojiri, Nagano 399-0781, Japan

E-mail: hiraga@po.mdu.ac.jp

Key words: side population, cancer stem cell, breast cancer, bone metastasis

\section{Introduction}

Despite the monoclonal origins, cancers are composed of a heterogeneous population of cells with different properties. It has recently been suggested that, within a given tumor, there exists a small population of cells with the capacity to behave like stem cells called cancer stem cells (CSCs) (1). This concept, which was first established in hematological malignancies (2), has been adapted for a growing number of solid tumors, including breast cancer $(1,3)$. CSCs are characterized by extensive self-renewal and proliferative capacity and resistance to chemotherapy and radiotherapy (1). These characteristic features play pivotal roles in the initiation, maintenance and relapse of tumors. Thus, CSCs are emerging as an important target for cancer therapy.

For identification and isolation of CSCs, several approaches have been proposed. In breast cancers, cell surface markers, such as CD24 and CD44, have been proved to be useful for the isolation of subsets enriched for breast CSCs $(1,3)$. High aldehyde dehydrogenase (ALDH) activity was also recently shown to identify the CSC fraction in breast cancer cells (4). An alternative approach is the side population (SP) phenomenon (5). Owing to the ability to exclude the DNA binding dye Hoechst 33342, SP cells are identified as a tail of dimly stained cells in dual wavelength fluorescent-activated cell sorter (FACS) analysis. Since the original discovery that SP cells in mouse bone marrow are highly enriched for longterm repopulating cells (6), SP cells have been identified in a variety of normal tissues and cancers, including breast cancer and shown to have stem cell-like properties $(5,7)$.

Bone is one of the most preferential metastatic target sites for breast cancer (8). It has been recently suggested that CSCs play an important role in mediating the metastasis of breast cancer $(9,10)$. However, it has yet to be elucidated whether CSCs contribute to the development of bone metastases.

In this study, we identified SP in the human breast cancer cell line MDA-MB-231 and characterized the SP cells as potential CSC-like cells. By taking advantage of the bone metastatic potential of MDA-MB-231 cells in a nude mouse model (11), we examined the ability of SP cells to metastasize to bone. 


\section{Materials and methods}

Reagents. Hoechst 33342, fumitremorgin C (FTC), verapamil and doxorubicin were purchased from Sigma-Aldrich (St. Louis, MO). B27 supplement, recombinant human fibroblast growth factor-2 (rhFGF-2) and recombinant human epidermal growth factor (rhEGF) were from Life Technologies Japan, Ltd. (Tokyo, Japan), BioVision, Inc. (Mountain View, CA), and R\&D Systems, Inc. (Minneapolis, MN), respectively. 5-Fluorouracil (5-FU) was from Kyowa Hakko Kirin Co., Ltd. (Tokyo, Japan). Matrigel (BD Matrigel Basement Membrane Matrix) was from Becton-Dickinson and Co. (Tokyo, Japan). All other chemicals used in this study were purchased from Sigma-Aldrich or Wako Pure Chemical Industries, Ltd. (Osaka, Japan) unless otherwise described.

Cell culture. Human breast cancer cell line MDA-MB-231 (American Type Culture Collection, Rockville, MD) was cultured in Dulbecco's modified Eagle's medium (DMEM; Life Technologies Japan, Ltd.) supplemented with $10 \%$ fetal bovine serum (FBS; Life Technologies Japan, Ltd.) and $100 \mu \mathrm{g} / \mathrm{ml}$ kanamycin sulfate (Meiji Seika Kaisha, Ltd., Tokyo, Japan). In some experiments, MDA-MB-231 cells were cultured in the presence of doxorubicin (10 or $30 \mathrm{nM})$ or 5-FU $(3,5$ or $10 \mu \mathrm{g} / \mathrm{ml})$ for 60 days and the surviving cells were used as doxorubicin- and 5-FU-resistant cells, respectively. The cells were maintained in a humidified atmosphere of $5 \%$ $\mathrm{CO}_{2}$ in air.

FACS analysis and cell sorting. SP analysis and sorting: The protocol was based on Goodell et al (6) with slight modifications. Briefly, MDA-MB-231 cells $\left(1 \times 10^{6}\right.$ cells $\left./ \mathrm{ml}\right)$ were incubated in Hanks' Balanced Salt Solutions (HBSS) supplemented with $2 \%$ FBS, $10 \mathrm{mM}$ HEPES, $1 \%$ penicillin/ streptomycin and $5 \mu \mathrm{g} / \mathrm{ml}$ Hoechst 33342 for $60 \mathrm{~min}$ at $37^{\circ} \mathrm{C}$ with intermittent mixing. In some experiments, cells were incubated with the Hoechst dye in the presence of $2 \mu \mathrm{M}$ FTC or $50 \mu \mathrm{M}$ verapamil. After incubation, cells were washed with cold medium and resuspended at $2 \times 10^{6}$ cells $/ \mathrm{ml}$, and propidium iodide was added to a final concentration of $2 \mu \mathrm{g} / \mathrm{ml}$ to discriminate dead cells. The gating of SP was based on negative controls in which FTC was used.

SP analysis of tumors from mice: MDA-MB-231 cells were inoculated into the mammary fat pad (5 million cells/ $0.1 \mathrm{ml}$ PBS $)$ or the left cardiac ventricle $(0.1$ million cells/ $0.1 \mathrm{ml}$ PBS) of athymic nude mice (female, 4-week-old, Japan SLC, Inc., Shizuoka, Japan) under anesthesia with pentobarbital $(0.05 \mathrm{mg} / \mathrm{g}$ body weight; Dainippon Sumitomo Pharma Co., Ltd., Osaka, Japan) (11). Four weeks after the inoculation, mammary tumors and bone metastases were dissected, minced with scalpels and strained through stainless steel mesh. The cells suspended with the culture medium were plated in $10 \mathrm{~cm}$ dishes and cultured overnight to remove dead cells and cell debris. Then, SP analysis was performed as described above.

CD24 and CD44 immunostaining: MDA-MB-231 cells were stained with rat monoclonal antibodies against CD24 (FITC-conjugated) and CD44 (PE-conjugated) or isotypematched IgG controls (all from eBiosciences, Inc., San Diego, CA).
Aldehyde dehydrogenase (ALDH) activity: ALDH enzymatic activity of MDA-MB-231 cells was examined using AldeFluor Kit (StemCell Technologies, Durham, NC) according to the manufacturer's protocol.

All FACS analysis and cell sorting were performed using a BD FACSVantage SE cell sorter (Becton-Dickinson and Co.). The data were analyzed by BD CellQuest Pro software (Becton-Dickinson and Co.).

Cell proliferation in monolayer. SP and NSP of MDA-MB-231 cells $(1,000$ cells/well/96-well plate) were plated in DMEM supplemented with $10 \%$ FBS and $100 \mu \mathrm{g} / \mathrm{ml}$ kanamycin sulfate and cultured for 24,72 and $96 \mathrm{~h}$. At the end of the culture, the cell proliferation was determined using Cell Proliferation Reagent WST-1 (Roche Diagnostics K.K., Tokyo, Japan) according to the manufacturer's protocol. The absorbance was measured using a microplate reader (Nippon Bio-Rad Laboratories, Tokyo, Japan) at a wavelength of $450 \mathrm{~nm}$.

Tumor-sphere formation. Tumor-sphere formation assay was performed as described previously with some modifications (12). SP and NSP isolated from MDA-MB-231 cells were plated in ultra-low attachment 6-well plates (Corning Japan K.K., Tokyo, Japan) at a density of 2,000 cells/well and cultured in DMEM/F12 (Sigma-Aldrich) containing 2\% B27 supplement, $40 \mathrm{ng} / \mathrm{ml}$ rhFGF-2 and $20 \mathrm{ng} / \mathrm{ml} \mathrm{rhEGF}$ for 10 days. The cells were replenished with fresh proliferation medium every 3 days. At the end of the culture, the number of tumorspheres of $>100 \mu \mathrm{m}$ in diameter was counted under a microscope and expressed as the number of tumor-spheres/ well.

Quantitative real-time reverse transcriptase-polymerase chain reaction $(q R T-P C R)$. Total RNA was isolated using RNeasy Plus Micro Kit (Qiagen K.K., Tokyo, Japan). Reverse transcription and real-time PCR was performed using One Step SYBR PrimeScript Plus RT-PCR Kit (Takara Bio Inc., Shiga, Japan) in DNA Engine Opticon system (Bio-Rad Laboratories, K.K., Tokyo, Japan) according to the manufacturer's protocol. Primer sequences were as follows: ATPbinding cassette transporters sub-family $\mathrm{G}$ member 2 (ABCG2), TGGCTTAGACTCAAGCACAGC/TCGTCC CTGCTTAGACATCC; ABCB1, ACAGAAAGCGAAGCA GTGGT/ATGGTGGTCCGACCTTTTC; glyceraldehyde-3phosphate dehydrogenase (GAPDH), AGCCACATCGCTC AGACAC/GCCCAATACGACCAAATCC. Melting curve analysis was performed to determine the melting temperature of the amplified product and to exclude undesired primer dimers. Each sample was run at least in triplicate. Quantification was normalized using GAPDH as a reference gene. Expression levels of the particular genes were indicated as fold increase compared with the control.

Microarray analysis. Total RNA was extracted from SP and NSP cells using RNeasy Plus Micro Kit. The RNAs were subjected to microarray full service (Biomatrix Research, Inc., Chiba, Japan) and assessed using GeneChip Human Gene 1.0 ST Array (Affymetrix, Inc., Santa Clara, CA). Array image was scanned and analyzed with GeneChip operating 
A

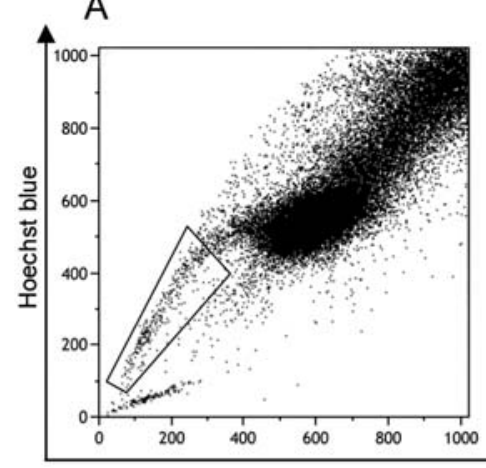

B

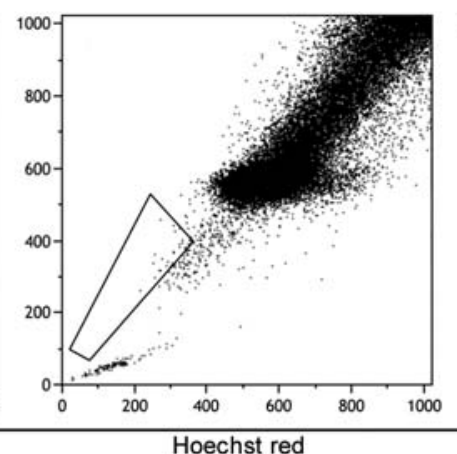

C

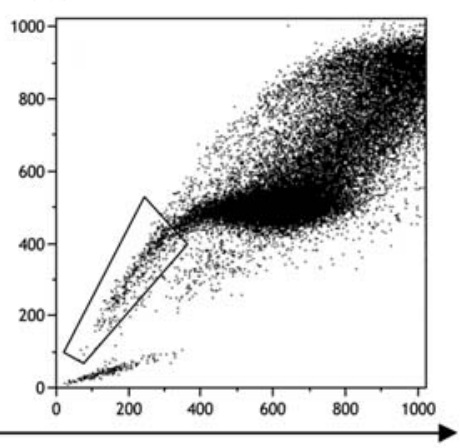

D

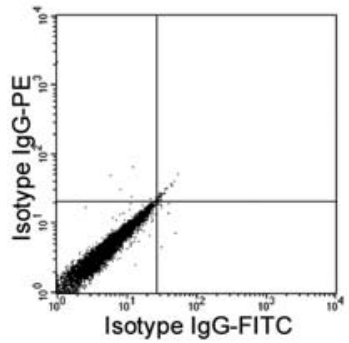

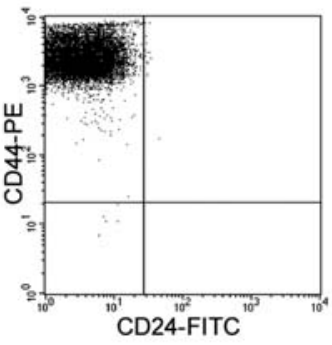

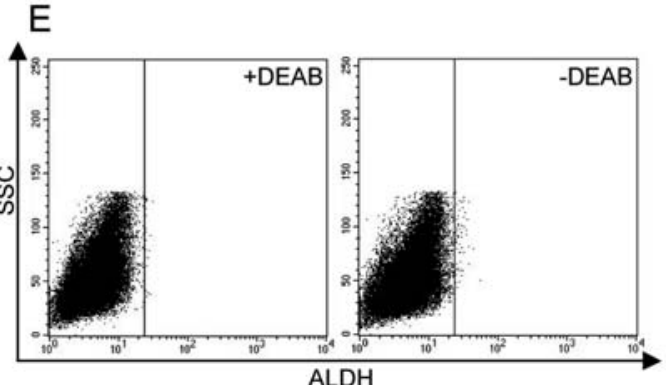

Figure 1. Expression of potential CSC markers in MDA-MB-231 human breast cancer cells. (A-C) Representative Hoechst 33342 dye staining profile in the absence (A) or presence of FTC (B) or verapamil (C). SPs are outlined. (D) CD24 and CD44 expression in MDA-MB-231 cells. The cells stained with isotype control IgGs are shown on the left. (E) ALDH activity of MDA-MB-231 cells in the presence (left) or absence (right) of diethylaminobenzaldehyde (DEAB), an inhibitor of ALDH.

software (Affymetrix, Inc.). Data mining was conducted using GeneSpring software (Agilent Technologies, Inc., Santa Clara, CA).

Animal experiments. Tumorigenesis in mammary fat pad: Under anesthesia with pentobarbital, SP and NSP cells $(1,000$ or 3,000 cells $/ 0.1 \mathrm{ml}$ matrigel) were injected into the mammary fat pad of athymic nude mice (female, 4-week-old, Japan SLC, Inc.). The size of orthotopic tumors was measured once a week using a caliper. The tumor volume was estimated by the following equation: tumor volume $\left(\mathrm{mm}^{3}\right)=$ (length) $\mathrm{x}$ (width) $)^{2} \times 0.5$.

Bone metastasis: SP and NSP cells $(10,000$ cells $/ 0.1 \mathrm{ml}$ PBS) were injected into the left cardiac ventricle of athymic nude mice (female, 4-week-old, Japan SLC, Inc.) under anesthesia with pentobarbital as described previously (11).

The number of mice used in each experiment is described in each figure. All animal experiments were reviewed and approved by the Animal Management Committee of Matsumoto Dental University.

$X$-ray analysis. Number and area of osteolytic lesions in the femora and tibiae were determined on radiographs as described previously (11). Data are shown as number of metastases/ mouse or lesion area $\left(\mathrm{mm}^{2}\right) /$ mouse.

Statistical analysis. Data are expressed as the mean \pm SEM. The data were analyzed by one-way ANOVA followed by Fisher's PLSD post-hoc test (StatView; SAS Institute, Inc., Cary, NC) for determination of differences between groups. Student's t-test or Welch's t-test was conducted when two groups were compared. P-values of $<0.05$ were considered significant.

\section{Results}

Expression of CSC markers in MDA-MB-231 human breast cancer cells. FACS analysis demonstrated that MDA-MB-231 cells after Hoechst 33342 staining were clearly separated into a fluorescence-negative tail of SP $(3.40 \pm 0.60 \%$, average of 10 independent experiments) and the brightly stained NSP (Fig. 1A). We also examined other potential CSC markers, CD24/CD44 expression and ALDH activity, in MDA-MB-231 cells. Most of the cells were CD24//CD44+ (Fig. 1D) and only a small number of the cells showed ALDH activity (Fig. 1E). Therefore, in the following experiments, we studied the SP of MDA-MB-231 cells as potential CSC-like cells in comparison to the NSP cells.

Characterization of SP cells in vitro. To examine the repopulation ability of the SP of MDA-MB-231 cells, SP and NSP cells were cultured for 2 weeks after cell sorting. Then, the cells were restained with Hoechst 33342 and reanalyzed by FACS. SP cells generated both SP and NSP with fraction size larger than or comparable to the original population (Fig. 2). The SP fraction generated from NSP was smaller than that from SP (Fig. 2).

$\mathrm{ABC}$ transporters, especially ABCG2, also known as breast cancer resistance protein (BCRP), and $\mathrm{ABCB} 1$, also known as multidrug resistance protein 1 (MDR1), have been implicated as being responsible for the SP phenotype $(13,14)$. Consistent with this notion, quantitative RT-PCR analysis showed that 


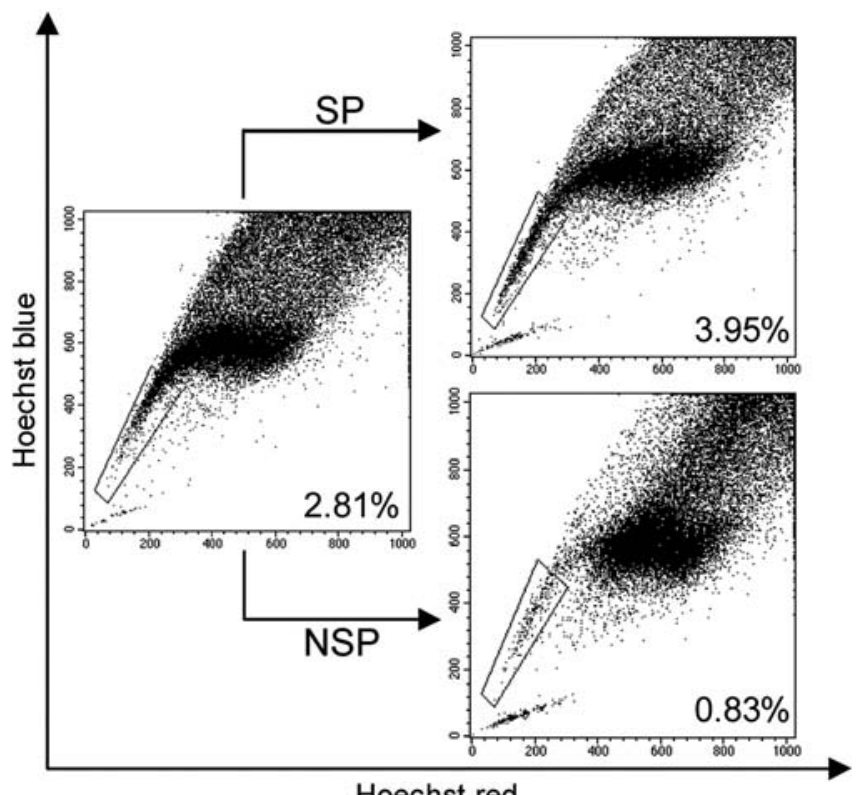

Hoechst red

Figure 2. Repopulation ability of the SP of MDA-MB-231 cells. SP and NSP sorted from MDA-MB-231 cells were cultured for 2 weeks. Then, the cells were restained with Hoechst dye and reanalyzed (right figures). Percentages given in the lower right corner refer to the relative abundance of SP cells.

ABCG2 mRNA expression was significantly higher in SP cells than in NSP cells (Fig. 3A), while ABCB1 mRNA was undetectable in both types. Furthermore, the SP fraction was markedly decreased in the presence of FTC, a specific inhibitor of $\mathrm{ABCG} 2$ (15), but not in the presence of verapamil, an inhibitor of $\mathrm{ABCB} 1$ (16) (Fig. 1B, C).

It has been reported that $\mathrm{ABC}$ transporters are involved in drug resistance in cancer cells (17). To determine the relationship between the SP phenotype and drug resistance, we tested the expression of $\mathrm{ABC}$ transporters and the SP abundance using MDA-MB-231 cells resistant to doxorubicin and 5-FU, the chemotherapeutic agents widely used for the treatment of breast cancer. ABCG 2 mRNA expression was significantly up-regulated in the doxorubicin-resistant cells in a dose-
A

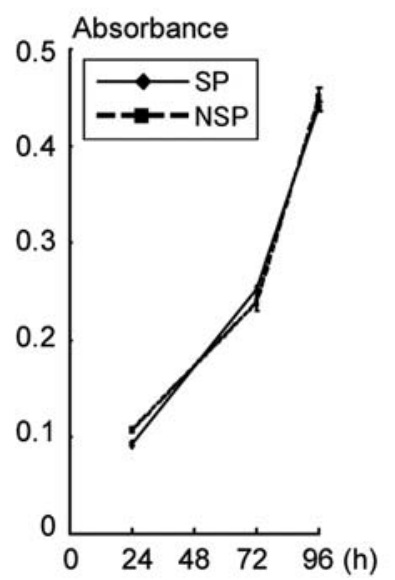

B

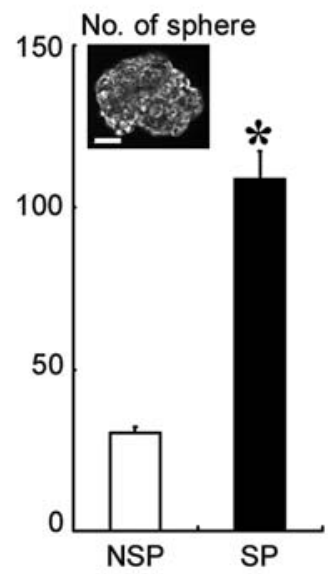

Figure 4. Cell proliferations of the SP of MDA-MB-231 cells in vitro. (A) Monolayer cell proliferation of SP and NSP cells determined by WST-1 assay. Data are shown as absorbance at $450 \mathrm{~nm}$. (B) Tumorsphere formation of SP and NSP cells in suspension culture. Data are shown as the number of tumorspheres per well. *Significantly different from NSP ( $<<0.05)$. Inset, representative phase contrast image of a tumorsphere formed by SP cells (scale bar, $50 \mu \mathrm{m}$ ).

dependent manner (Fig. 3B), whereas ABCB1 mRNA was not detected in any of the drug-resistant cells. Furthermore, the SP abundance was increased in the doxorubicin-resistant cells (Fig. 3C). These changes were not observed in the 5-FUresistant cells (data not shown).

We then examined the cell proliferation of SP in vitro. Monolayer cell proliferation of SP cells was similar to that of NSP cells (Fig. 4A). In contrast, the tumorsphere formation in suspension culture was significantly enhanced in SP cells (Fig. 4B).

Tumorigenic and metastatic potential of SP cells in nude mice. To examine the tumorigenicity in vivo, the SP of MDA-MB231 cells were inoculated into the mammary fat pad in nude

A

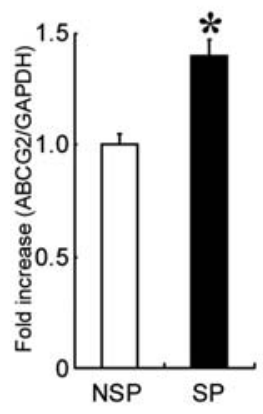

B

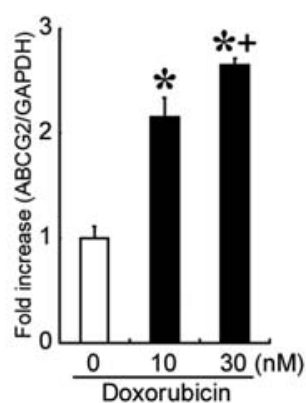

C

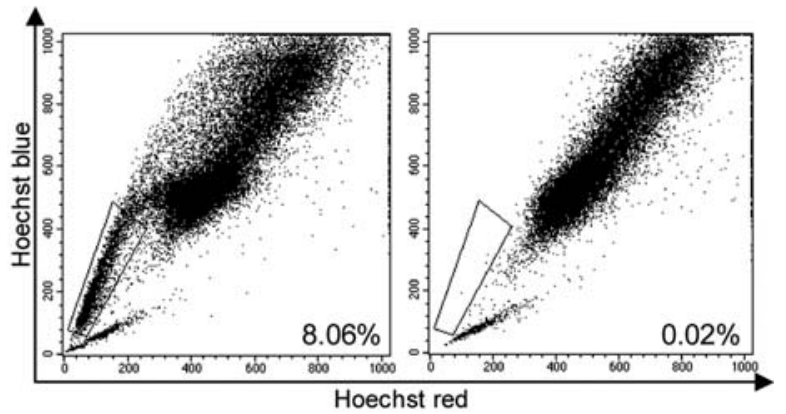

Figure 3. SP phenotype and ABCG2 expression. (A) Relative ABCG2 mRNA expression in SP and NSP of MDA-MB-231 cells determined by real-time RT-PCR. Data are shown as fold increase compared with NSP. *Significantly different from NSP (p<0.05). (B) Relative ABCG2 mRNA expression in doxorubicin-resistant MDA-MB-231 cells determined by real-time RT-PCR. Data are shown as fold increase compared with doxorubicin-untreated (0 nM) control cells. "Significantly different from the control $(\mathrm{p}<0.05)$, ${ }^{+}$significantly different from doxorubicin $(10 \mathrm{nM})(\mathrm{p}<0.05)$. (C) Representative Hoechst 33342 dye staining profile of doxorubicin (10 nM)-resistant MDA-MB-231 cells in the absence (left) or presence of FTC (right). Percentages given in the lower right corner refer to the relative abundance of SP cells. 
Table I. Tumorigenicity of the SP of MDA-MB-231 cells in nude mice.

\begin{tabular}{llc}
\hline \multirow{2}{*}{ Inoculated cell no. } & \multicolumn{2}{c}{ Tumor formation rate } \\
\cline { 2 - 3 } & NSP & SP \\
\hline 1,000 & $3 / 11$ & $5 / 11$ \\
3,000 & $5 / 13$ & $6 / 13$ \\
\hline
\end{tabular}

SP and NSP cells (1,000 or 3,000 cells/mouse) were inoculated into the mammary fat pad in nude mice $(\mathrm{n}=11$ or 13$)$. Tumor formation was assessed at 8 weeks after the cell inoculation.

mice. Tumor formation rates at 8 weeks after the cell inoculation were similar between SP and NSP cells (Table I). However, the tumor growth of SP cells was significantly accelerated compared with that of NSP cells (Fig. 5A).

The bone-metastatic potential of SP cells was next examined by inoculating the cells into the left cardiac ventricle in nude mice. Radiographic analysis demonstrated that both SP and NSP cells developed osteolytic bone metastases (Fig. 5B). The incidences of metastasis were similar between SP and NSP (NSP: 10/15, SP: 9/15, the number of mice with metastases/total mice). There was no significant difference in the lesion number and area (Fig. 5B, C).

SP abundance in bone metastases. We examined whether SP is increased in bone metastases by inoculating parental MDA-MB-231 cells into nude mice. The SP abundance in the tumor cells in bone metastases was comparable to that in the mammary tumors and in parental MDA-MB-231 cells in culture (Fig. 6).

Gene expression profile of SP cells. We finally performed a comprehensive analysis of gene expression differences between SP and NSP cells. The microarray data demon-

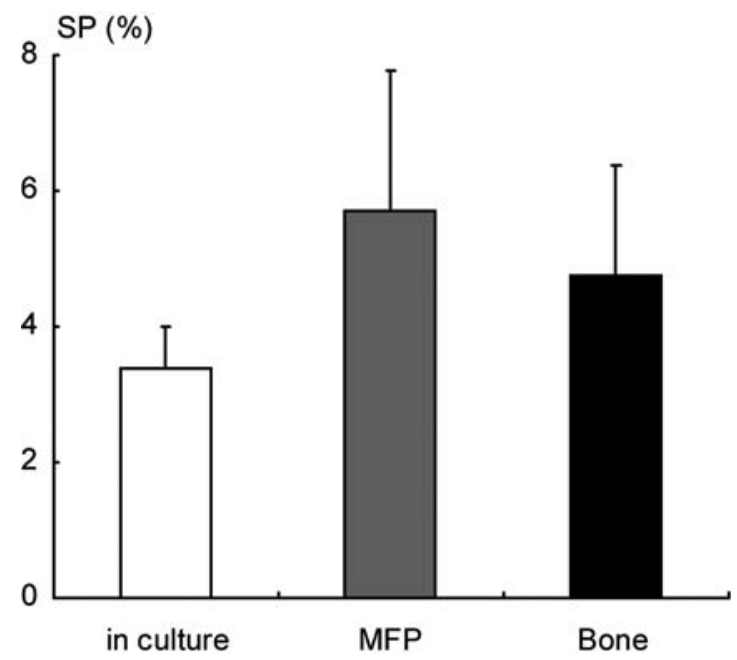

Figure 6. SP abundance in bone metastases of parental MDA-MB-231 cells. The abundance of SP (\%) in tumor cells isolated from the tumors in mammary fat pad (MFP) and in bone ( $\mathrm{n}=9$ /group) is shown. The SP abundance in parental MDA-MB-231 cells in culture is also shown as a control (left bar).

strated that 44 genes showed more than 1.5-fold up-regulation and 29 genes showed a $>1.5$-fold down-regulation (Table II). However, no up-regulation of known stem cell markers, potential cancer stem cell markers and metastasis-related genes was found in SP cells.

\section{Discussion}

Although several markers have been proposed to identify CSCs, the definitive markers have not been established in any types of CSC. In breast cancers, the cell surface markers $\mathrm{CD} 24^{- \text {llow/CD44 }}{ }^{+}$, high ALDH activity and SP are considered to be potential markers for CSCs $(1,3-5,7)$. However, it is curious that the cell populations isolated using these markers do not always overlap (4). Consistent with these results, our data show that most MDA-MB-231 cells were CD24-/CD44+,
A

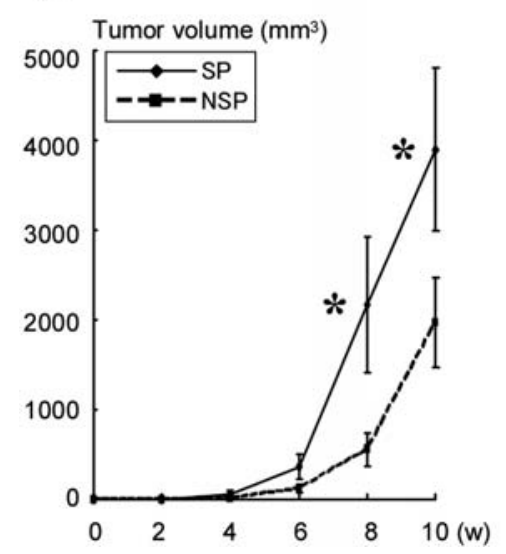

B

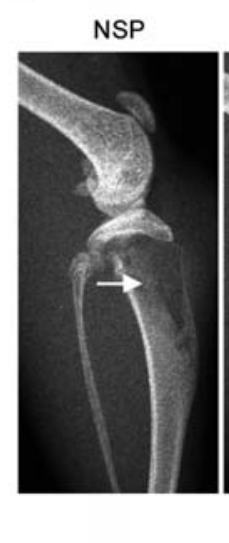

C

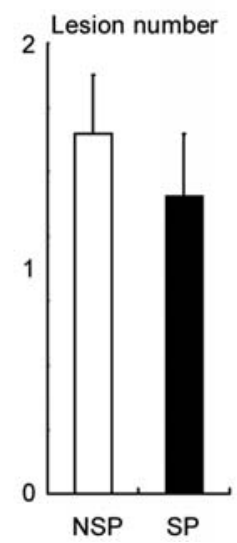

Figure 5. Tumorigenic and metastatic potential of the SP of MDA-MB-231 cells. (A) Tumor growth of SP and NSP cells (3,000 cells/mouse) in the orthotopic mammary fat pad in nude mice ( $\mathrm{n}=6$ /group). *Significantly different from NSP $(\mathrm{p}<0.05)$. (B and C) Development of bone metastases in nude mice. SP and NSP cells $(10,000$ cells/mouse) were inoculated through the left cardiac ventricle ( $n=10 / N S P, n=9 / S P)$. (B) Representative radiographs of the osteolytic lesions in hindlimbs at 6 weeks after the cell inoculation (arrows, osteolytic bone metastases). (C) Six weeks after the cell inoculation, the lesion number (left) and area (right) of osteolytic bone metastases were measured on radiographs. Data are shown as lesion number/mouse or lesion area (mm²)/mouse. 
Table II. Microarray analysis of the SP and NSP isolated from MDA-MB-231 cells.

\begin{tabular}{|c|c|c|c|}
\hline Gene symbol & Gene description & Fold change & Status \\
\hline MIPEP & Mitochondrial intermediate peptidase & 2.66 & Up \\
\hline EIF4A2 & Eukaryotic translation initiation factor $4 \mathrm{~A}$, isoform 2 & 2.5 & Up \\
\hline SNORA4 & Small nucleolar RNA, H/ACA box 4 & 2.44 & Up \\
\hline TISP43 & Similar to mCG22736 & 2.01 & Up \\
\hline FAM99A & Family with sequence similarity 99 , member A & 1.93 & Up \\
\hline KIR2DS5 & $\begin{array}{l}\text { Killer cell immunoglobulin-like receptor, two domains, } \\
\text { short cytoplasmic tail, } 5\end{array}$ & 1.93 & Up \\
\hline SNORD52 & Small nucleolar RNA, C/D box 52 & 1.83 & Up \\
\hline SNORA1 & Small nucleolar RNA, H/ACA box 1 & 1.79 & Up \\
\hline C15orf51 & Chromosome 15 open reading frame 51 & 1.78 & Up \\
\hline LOC100130904 & Similar to CD177 molecule & 1.77 & Up \\
\hline IL28A & Interleukin 28A (interferon, $\lambda 2$ ) & 1.74 & Up \\
\hline CXorf42 & Chromosome $\mathrm{X}$ open reading frame 42 & 1.69 & Up \\
\hline LOC100132178 & Similar to hCG2008275 & 1.69 & Up \\
\hline HIST2H2BE & Histone cluster $2, \mathrm{H} 2 \mathrm{be}$ & 1.68 & Up \\
\hline LOC283588 & NA & 1.66 & Up \\
\hline LOC100129391 & $\begin{array}{l}\text { Similar to Zinc finger (CCCH type), RNA-binding motif } \\
\text { and serine/arginine rich } 2\end{array}$ & 1.65 & Up \\
\hline LOC100128830 & NA & 1.64 & Up \\
\hline FLJ31958 & NA & 1.63 & Up \\
\hline C3orf47 & Chromosome 3 open reading frame 47 & 1.63 & Up \\
\hline WIF1 & WNT inhibitory factor 1 & 1.63 & Up \\
\hline GAGE13 & $\mathrm{G}$ antigen 13 & 1.62 & Up \\
\hline MIRN21 & microRNA 21 & 1.62 & Up \\
\hline DEXI & Dexamethasone-induced transcript & 1.62 & Up \\
\hline TAF12 & NA & 1.61 & Up \\
\hline MGC39584 & Hypothetical gene supported by BC029568 & 1.59 & Up \\
\hline C4orf11 & Chromosome 4 open reading frame 11 & 1.59 & Up \\
\hline FRMPD2 & FERM and PDZ domain containing 2 & 1.59 & Up \\
\hline LOC100129420 & Similar to hCG1990547 & 1.59 & Up \\
\hline OR4C13 & Olfactory receptor, family 4 , subfamily $\mathrm{C}$, member 13 & 1.57 & Up \\
\hline SNORD75 & Small nucleolar RNA, C/D box 75 & 1.56 & Up \\
\hline KCNIP1 & $\mathrm{Kv}$ channel interacting protein 1 & 1.55 & Up \\
\hline LRMP & Lymphoid-restricted membrane protein & 1.55 & Up \\
\hline C21orf94 & Chromosome 21 open reading frame 94 & 1.54 & Up \\
\hline SNORA16B & Small nucleolar RNA, H/ACA box 16B & 1.54 & Up \\
\hline ABCG2 & ATP-binding cassette, sub-family G (WHITE), member 2 & 1.54 & Up \\
\hline TTC30B & Tetratricopeptide repeat domain $30 \mathrm{~B}$ & 1.53 & Up \\
\hline SNORD25 & Small nucleolar RNA, C/D box 25 & 1.53 & Up \\
\hline FLJ30064 & Hypothetical protein LOC644975 & 1.53 & Up \\
\hline HOXD10 & Homeobox D10 & 1.53 & Up \\
\hline OR4D1 & Olfactory receptor, family 4 , subfamily $\mathrm{D}$, member 1 & 1.52 & Up \\
\hline RP11-114H20.1 & Hypothetical LOC401589 & 1.52 & Up \\
\hline C1orf62 & Chromosome 1 open reading frame 62 & 1.52 & Up \\
\hline C4orf38 & Chromosome 4 open reading frame 38 & 1.51 & Up \\
\hline SLFN12L & Schlafen family member 12-like & 1.51 & Up \\
\hline OR4N4 & Olfactory receptor, family 4 , subfamily $\mathrm{N}$, member 4 & 1.51 & Up \\
\hline HSPC072 & NA & 2.61 & Down \\
\hline C20orf69 & Chromosome 20 open reading frame 69 & 2.05 & Down \\
\hline GABRE & $\gamma$-aminobutyric acid (GABA) A receptor, $\varepsilon$ & 1.99 & Down \\
\hline
\end{tabular}


Table II. Continued.

\begin{tabular}{|c|c|c|c|}
\hline Gene symbol & Gene description & Fold change & Status \\
\hline SLCO1B3 & Solute carrier organic anion transporter family, member 1B3 & 1.93 & Down \\
\hline LOC221442 & NA & 1.88 & Down \\
\hline LCE2C & Late cornified envelope $2 \mathrm{C}$ & 1.88 & Down \\
\hline CATSPER2P1 & Cation channel, sperm associated 2 pseudogene 1 & 1.76 & Down \\
\hline LOC152118 & NA & 1.74 & Down \\
\hline SNORD41 & Small nucleolar RNA, C/D box 41 & 1.74 & Down \\
\hline CDRT1 & CMT1A duplicated region transcript 1 & 1.73 & Down \\
\hline LOC51336 & Mesenchymal stem cell protein DSCD28 & 1.69 & Down \\
\hline ID2 & $\begin{array}{l}\text { Inhibitor of DNA binding 2, dominant negative helix- } \\
\text { loop-helix protein }\end{array}$ & 1.69 & Down \\
\hline LOC441233 & Hypothetical gene supported by AK128010 & 1.68 & Down \\
\hline SNORA15 & Small nucleolar RNA, H/ACA box 15 & 1.66 & Down \\
\hline FLJ27243 & NA & 1.64 & Down \\
\hline MMP1 & Matrix metallopeptidase 1 (interstitial collagenase) & 1.63 & Down \\
\hline DOC2B & Double C2-like domains, $ß$ & 1.61 & Down \\
\hline OR7G1 & Olfactory receptor, family 7 , subfamily G, member 1 & 1.6 & Down \\
\hline OR1S1 & Olfactory receptor, family 1 , subfamily $\mathrm{S}$, member 1 & 1.6 & Down \\
\hline SNORD38B & Small nucleolar RNA, C/D box 38B & 1.59 & Down \\
\hline MARCH11 & Membrane-associated ring finger (C3HC4) 11 & 1.59 & Down \\
\hline RBMY2EP & $\begin{array}{l}\text { RNA binding motif protein, Y-linked, family } 2 \text {, } \\
\text { member E pseudogene }\end{array}$ & 1.57 & Down \\
\hline N6AMT1 & N-6 adenine-specific DNA methyltransferase 1 (putative) & 1.54 & Down \\
\hline ENTPD3 & Ectonucleoside triphosphate diphosphohydrolase 3 & 1.52 & Down \\
\hline KIR2DL3 & $\begin{array}{l}\text { Killer cell immunoglobulin-like receptor, two domains, } \\
\text { long cytoplasmic tail, } 3\end{array}$ & 1.52 & Down \\
\hline GOLGA6 & Golgi autoantigen, golgin subfamily a, 6 & 1.52 & Down \\
\hline LOC646934 & Similar to golgin-like protein & 1.52 & Down \\
\hline LOC441956 & Similar to cDNA sequence BC021523 & 1.51 & Down \\
\hline VCAN & Versican & 1.51 & Down \\
\hline
\end{tabular}

Up- (>1.5-fold) and down-regulated (>1.5-fold) genes in the SP of MDA-MB-231 cells are shown. NA, not applicable.

whereas a very small population of MDA-MB-231 cells possessed ALDH activity and a few percent of the cells entered the SP fraction. Extensive studies are awaited to determine the generalized markers for breast CSCs.

SP cells have been identified in a variety of human cancers and cancer cell lines and shown to have CSC-like properties $(5,7)$. The SP of MDA-MB-231 cells also displayed CSC-like characteristics, such as enhanced tumor-sphere formation in culture and increased tumor growth in the mammary fat pad in nude mice. However, there are some reports indicating that SP does not completely define CSC-like cells $(18,19)$. They demonstrated that, as well as SP cells, NSP cells gave rise to a significant proportion of SP cells. On the basis of stem cell physiology, SP cells should give rise to SP and NSP cells by means of asymmetric cell division, whereas NSP cells should not have this potential. In our study, the NSP of MDA-MB-231 cells also gave rise to SP, although the population was smaller than the SP generated from SP.
Furthermore, the NSP developed tumors in mammary fat pad in nude mice at a similar rate to the SP. These results collectively suggest that the SP of MDA-MB-231 cells possesses some of the CSC-like properties but does not completely meet the criteria for CSCs.

It has been suggested that the SP phenotype is conferred by the expression of $\mathrm{ABC}$ transporters, especially $\mathrm{ABCB} 1$ and ABCG2 $(13,14)$. The SP fraction of MDA-MB-231 cells was eliminated in the presence of an ABCG2-specific inhibitor FTC. ABCG2 mRNA expression was significantly upregulated in SP cells compared with that in NSP cells. These results suggest that $\mathrm{ABCG} 2$ is responsible, at least in part, for the SP phenotype of MDA-MB-231 cells.

CSCs are thought to be resistant to chemotherapy. One mechanism by which this may arise is through the expression of ABC transporters. In this respect, SP cells share similar properties with CSCs. The doxorubicin-resistant MDA-MB231 cells exhibited higher ABCG2 expression and increased 
SP fraction, suggesting a relationship between the SP phenotype and drug resistance. In contrast, these changes were not found in the 5-FU-resistant cells. Since ABC transporters responsible for the efflux of each chemotherapeutic agent are variable (17), it may be possible that 5-FU is effluxed by $\mathrm{ABC}$ transporters other than $\mathrm{ABCB} 1$ and $\mathrm{ABCG} 2$ in MDAMB-231 cells.

The development of distant metastases is the major cause of death in patients with breast cancer. However, the role of CSCs in multistage cancer progression, particularly with respect to metastasis, has not been well defined. Breast cancer cells with $\mathrm{CD} 24-/ \mathrm{CD} 44^{+}$subpopulation have been shown to have higher invasive properties (20). ALDH-positive populations of mammary carcinoma cell lines also display increased invasive characteristics (10). These findings suggest that CSCs possess higher metastatic potential. Furthermore, Balic et al have revealed an increase in $\mathrm{CD} 24 / \mathrm{CD} 44^{+}$cancer cells disseminated in bone marrow in breast cancer patients (21), suggesting that CSCs may have high metastatic potential to bone. Meanwhile, there are some reports showing that CSC phenotype alone is not enough to determine metastasis $(1,20)$. Our results show that SP cells did not exhibit up-regulation of metastasis-related genes and did not cause increased metastases to bone. These results suggest that CSCs may not have higher metastatic potential to bone. Experiments using the definitive CSCs have to be performed to determine the role of CSCs in cancer metastasis.

We also found that the SP abundance in bone metastases in nude mice is comparable to those in mammary fat pad and in parental MDA-MB-231 cells in culture. However, the data do not exclude the possibility that SP cells may have a higher metastatic potential than NSP cells, because the results including ours indicate that the ratio of SP to NSP cells reached a steady state within a few weeks (22). Thus, it is possible that cancer cells initially metastasize as SP and then change their phenotype following limited differentiation at their new site.

It has been argued that the phenotypic differences between SP and NSP cells are a consequence of damage induced by the Hoechst dye, which is retained longer in NSP cells. Christgen et al proposed that the Hoechst dye directly affects the expression of stemness-related genes (23). In our study, monolayer cell proliferation was similar between SP and NSP. The expression of stem cell genes was also unaffected in NSP cells compared to that in SP cells. Thus, it is unlikely that the Hoechst dye directly caused the difference between SP and NSP in MDA-MB-231 cells, although it is difficult to exclude the possibility.

In conclusion, our results suggest that the SP of MDAMB-231 cells possesses some of the properties of CSCs but does not have a higher metastatic potential to bone. However, since definite markers for CSCs have not been established yet, further studies are required to determine the bone metastatic potential of CSCs.

\section{Acknowledgements}

This study was supported by grants in aid from the Ministry of Education, Culture, Sports, Science and Technology, Japan (to T.H. and H.N.).

\section{References}

1. Visvader JE and Lindeman GJ: Cancer stem cells in solid tumours: accumulating evidence and unresolved questions. Nat Rev Cancer 8: 755-768, 2008.

2. Bonnet D and Dick JE: Human acute myeloid leukemia is organized as a hierarchy that originates from a primitive hematopoietic cell. Nat Med 3: 730-737, 1997.

3. Al-Hajj M, Wicha MS, Benito-Hernandez A, Morrison SJ and Clarke MF: Prospective identification of tumorigenic breast cancer cells. Proc Natl Acad Sci USA 100: 3983-3988, 2003.

4. Ginestier C, Hur MH, Charafe-Jauffret E, et al: Aldh1 is a marker of normal and malignant human mammary stem cells and a predictor of poor clinical outcome. Cell Stem Cell 1: 555-567, 2007.

5. Wu C and Alman BA: Side population cells in human cancers. Cancer Lett 268: 1-9, 2008.

6. Goodell MA, Brose K, Paradis G, Conner AS and Mulligan RC: Isolation and functional properties of murine hematopoietic stem cells that are replicating in vivo. J Exp Med 183: 1797-1806, 1996.

7. Nakanishi T, Chumsri S, Khakpour N, et al: Side-population cells in luminal-type breast cancer have tumour-initiating cell properties, and are regulated by HER 2 expression and signalling. Br J Cancer 102: 815-826, 2010.

8. Mundy G: Metastasis to bone: causes, consequences and therapeutic opportunities. Nat Rev Cancer 2: 584-593, 2002.

9. Croker AK, Goodale D, Chu J, et al: High aldehyde dehydrogenase and expression of cancer stem cell markers selects for breast cancer cells with enhanced malignant and metastatic ability. J Cell Mol Med 13: 2236-2252, 2008.

10. Charafe-Jauffret E, Ginestier C, Iovino F, et al: Breast cancer cell lines contain functional cancer stem cells with metastatic capacity and a distinct molecular signature. Cancer Res 69: 1302-1313, 2009.

11. Hiraga $T$ and Nakamura H: Imatinib mesylate suppresses bone metastases of breast cancer by inhibiting osteoclasts through the blockade of c-Fms signals. Int J Cancer 124: 215-222, 2009.

12. Hansford LM, McKee AE, Zhang L, et al: Neuroblastoma cells isolated from bone marrow metastases contain a naturally enriched tumor-initiating cell. Cancer Res 67: 11234-11243, 2007.

13. Bunting KD, Zhou S, Lu T and Sorrentino BP: Enforced Pglycoprotein pump function in murine bone marrow cells results in expansion of side population stem cells in vitro and repopulating cells in vivo. Blood 96: 902-909, 2000.

14. Zhou S, Schuetz JD, Bunting KD, et al: The ABC transporter Bcrp1/ABCG2 is expressed in a wide variety of stem cells and is a molecular determinant of the side-population phenotype. Nat Med 7: 1028-1034, 2001.

15. Rabindran SK, Ross DD, Doyle LA, Yang W and Greenberger LM: Fumitremorgin $C$ reverses multidrug resistance in cells transfected with the breast cancer resistance protein. Cancer Res 60: 47-50, 2000.

16. Couture L, Nash JA and Turgeon J: The ATP-binding cassette transporters and their implication in drug disposition: a special look at the heart. Pharmacol Rev 58: 244-258, 2006.

17. Dean M: ABC transporters, drug resistance, and cancer stem cells. J Mammary Gland Biol Neoplasia 14: 3-9, 2009.

18. Lichtenauer UD, Shapiro I, Geiger K, et al: Side population does not define stem cell-like cancer cells in the adrenocortical carcinoma cell line NCI H295R. Endocrinology 149: 1314-1322, 2008.

19. Mitsutake N, Iwao A, Nagai K, et al: Characterization of side population in thyroid cancer cell lines: cancer stem-like cells are enriched partly but not exclusively. Endocrinology 148: 1797-1803, 2007.

20. Sheridan C, Kishimoto H, Fuchs RK, et al: CD44 ${ }^{+} \mathrm{CD} 24^{-}$breast cancer cells exhibit enhanced invasive properties: an early step necessary for metastasis. Breast Cancer Res 8: R59, 2006.

21. Balic M, Lin H, Young L, et al: Most early disseminated cancer cells detected in bone marrow of breast cancer patients have a putative breast cancer stem cell phenotype. Clin Cancer Res 12: 5615-5621, 2006.

22. Hirschmann-Jax C, Foster AE, Wulf GG, et al: A distinct 'side population' of cells with high drug efflux capacity in human tumor cells. Proc Natl Acad Sci USA 101: 14228-14233, 2004.

23. Christgen M, Geffers R, Ballmaier M, et al: Down-regulation of the fetal stem cell factor SOX17 by H33342: a mechanism responsible for differential gene expression in breast cancer side population cells. J Biol Chem 285: 6412-6418, 2010. 